\title{
Propuesta para la inserción laboral de docentes en contextos de alta vulnerabilidad social
}

\section{Proposal for Labor Market Integration of Teachers in Contexts of High Social Vulnerability}

\author{
Magally Gutiérrez Gutiérrez ${ }^{1}$ \\ Universidad de Costa Rica \\ San José, Costa Rica \\ magallygu05@gmail.com
}

Montserrat Vindas Cordero ${ }^{2}$

Centro de Investigación y Docencia en Educación de la Universidad Nacional

Heredia, Costa Rica

montsevincor@hotmail.com

Recibido 05 de mayo de 2010 • Aceptado 31 de agosto de 2010

\begin{abstract}
Resumen. Este artículo tiene como objetivo socializar una propuesta de inserción laboral de docentes en contextos de alta vulnerabilidad social ${ }^{3}$, producto de la investigación realizada en una escuela de atención prioritaria del cantón central de Heredia ${ }^{4}$, en la cual se exploró el proceso de inserción laboral que se desarrolla con los docentes, con la intención de que estos respondan a la realidad comunal, familiar y estudiantil de una población inmersa en una dinámica de riesgo social. La investigación que sustenta la propuesta parte de un enfoque cualitativo, ya que el proceso de diagnóstico no pretende dar respuestas generalizables a otros centros educativos en contextos similares, sino lograr una aproximación exploratoria a la realidad docente y a su proceso de inserción en instituciones con alta vulnerabilidad social. Por tanto, el artículo, si bien plantea la intención de compartir esta experiencia, no pretende unificar prácticas de inserción, sino más bien ser un insumo en la realización de procesos similares.
\end{abstract}

Palabras claves. Vulnerabilidad social, inserción laboral, permanencia institucional.

\footnotetext{
Licenciada en Educación Preescolar y académica de la Universidad de Costa Rica. Magíster en Formación Formadores de Docentes de Educación Primaria.

2 Licenciada en Educación Preescolar y académica del Centro de Investigación y Docencia en Educación (CIDE) de la Universidad Nacional, Costa Rica. Magíster en Formación de Formadores de Docentes de Educación Primaria.

3 Entendiéndose como contexto de alta vulnerabilidad social el planteamiento realizado por Sojo (2003) que lo define como comunidades urbano marginales situadas en zonas catalogadas por el gobierno costarricense como de atención prioritaria, las cuales presentan el mayor atraso social y económico del país, con altos índices de violencia, ocio, desempleo y drogadicción.

$4 \quad$ La institución educativa es de carácter público, y pertenece al Circuito 02 de esta Provincia.
} 
Abstract. The purpose of this paper is to share a proposal for teacher's labor market integration in contexts of high social ${ }^{5}$ vulnerability. This paper is the result of a research conducted in a priority attention primary school ${ }^{6}$ of the central canton of Heredia ${ }^{7}$. It explored the labor market integration process of teachers, considering the community, family and student reality of a population social risk. The research that supports this proposal is based on a qualitative approach, since the diagnosis process is not intended to provide answers that could be commonly applied to other education centers in similar contexts, but to make an exploratory approach of teachers' reality and their integration process into education institutions of high social vulnerability. Therefore, although this paper intends to share this experience, it does not aim to unify integration practices, but to be an input in carrying out similar processes.

Keywords. Social vulnerability, labor market integration, institutional permanence.

(...) la calidad de la educación será posible si hay educadoras con capacidad de re-mirar sus prácticas y de re-pensarlas para revalorizar la propia cultura, retomando las fortalezas humanas, naturales y culturales (...)

Victoria Peralta (2007)

\section{Introducción}

La investigación "Análisis de procesos de inserción laboral docente en centros educativos ubicados en contextos de alta vulnerabilidad social: una experiencia realizada en la Escuela de Atención Prioritaria de la provincia de Heredia", se llevó a cabo durante el último trimestre del 2009. Se espera que este estudio constituya un aporte al enriquecimiento de la educación costarricense, como parte de la Maestría Centroamericana en Formación de Formadores de Docentes de Educación Primaria, del Centro de Investigación y Docencia en Educación, en la División de Educación Rural (DER) de la Universidad Nacional.

Este proyecto surge de la inquietud de las investigadoras, quienes, a partir de su experiencia como docentes en centros educativos ubicados en diversos contextos geográficos, sociales y culturales, visualizan la necesidad de analizar el proceso de inserción laboral en estos.

El trabajo en estas comunidades requiere desarrollar una sensibilidad particular hacia la problemática social, implica más flexibilidad y contar con las herramientas que ayuden a unificar el proceso de enseñanza con el entorno. La realidad de los educandos y de sus familias demanda respuestas que deben atender otro tipo de necesidades emergentes, como el vandalismo, la delincuencia, la violencia en el hogar, entre otros. Estas necesidades van más allá de procesos cognitivos y de aplicación de adecuaciones curriculares, que bien se abordan en la formación docente universitaria. Sin embargo, eso no es suficiente. Arce, Gamboa, Glock, Paifel y Sánchez

\footnotetext{
The concept of high social vulnerability is understood based on Sojo's approach (2003), which defines it as marginal urban communities in areas considered by the Costa Rican government as priority areas with the greatest social, economic backwardness in the country, and high rates of violence, leisure, unemployment and drug addiction.

6 Translator's note: The Costa Rican education system is composed of primary education (1st-6th grade) and secondary education (7th-11th grade).

7 A public primary school in the circuit 02 of the Province of Heredia.
} 
(2001) resaltan que el docente no solo debe poseer vocación, sino también ser consciente de la problemática del entorno. Por tanto, la propuesta de inserción que este artículo reconstruye viene a contribuir en esta carencia dentro del sistema educativo costarricense.

Respondiendo a la afirmación anterior, el Consejo Superior de Educación (1995) señala que los docentes en los contextos de vulnerabilidad social deben responder al mejoramiento de la calidad de vida de cada individuo, y buscar en los programas educativos una esperanza de vida, una construcción social que responda a lo que él o ella se proponga ser y no lo que la sociedad necesariamente le establezca. Por tanto, las condiciones educativas de estos centros deben visualizarse como instancias donde se maximicen las oportunidades, para que el individuo descubra la necesidad de su transformación social. Todo ello se fundamenta en lo que establece el Programa Estado de la Nación en Desarrollo Humano Sostenible (2008), en el Informe Estado de la Educación Costarricense 2, cuando define que el “(...) papel del docente es fundamental en una sociedad, por lo que los educadores tienen el potencial de influir en los niños y jóvenes en su proceso de desarrollo y facilitarles el acceso a oportunidades presentes y futuras para vivir vidas más gratificantes y promover el desarrollo humano del país" (p. 123).

Es así como los docentes que laboran en contextos de alta vulnerabilidad social deben participar en acciones de capacitación que los apoyen en su proceso de inserción laboral, para cumplir de este modo con el compromiso de desempeñarse de manera eficiente. Por tanto, se pretende que al elaborar lineamientos y estrategias para la inserción laboral docente se logre subsanar una debilidad en el accionar del Ministerio de Educación Pública.

\section{Planteamiento de la investigación}

A partir de las ideas planteadas, se considera pertinente realizar un diagnóstico exploratorio y una revisión de las características sociales y profesionales que poseen los educadores que se desempeñan en centros educativos ubicados en estos contextos. El proceso investigativo busca identificar si estas características responden a las demandas pedagógicas de la realidad institucional y cómo se fortalecen en los procesos de inserción laboral. Para ello, se parte de la premisa de que todo docente debe contar con al menos tres características fundamentales: sensibilidad, flexibilidad y conocimiento.

Para orientar el desarrollo del proceso investigativo se formuló el siguiente problema:

¿Cuál es el proceso de inserción laboral docente que se desarrolla en los centros educativos ubicados en contextos de alta vulnerabilidad social?

Y se define como objetivo general el siguiente:

Explorar el proceso de inserción laboral que se desarrolla con los docentes de centros educativos en contextos de alta vulnerabilidad social, para fundamentar lineamientos y estrategias que faciliten su labor y permanencia en este tipo de contextos. 


\section{Sustento teórico}

Entre los referentes teóricos que dan sustento al estudio se presenta el concepto de inserción laboral, definido según González, Araneda, Hernández y Lorca (2005) como un procedimiento que incorpora en la práctica la relación entre las características personales, el manejo técnico y el conocimiento del contexto institucional. Estos autores asumen la necesidad de un programa sistemático de inducción al profesor principiante, y demanda la preparación y el entrenamiento de profesores que acompañen al docente nuevo en su fase inicial. En la relación de estos tres tipos de características, el docente nuevo en el contexto, o en su desarrollo profesional, acude a su caudal de experiencias, conocimientos, habilidades y competencias derivadas de su formación inicial.

Además, se formula la definición de contextos de alta vulnerabilidad social, sustentada por Sojo (2003) y entendida como las comunidades urbano marginales situadas en zonas catalogadas por el gobierno costarricense como de atención prioritaria, las cuales presentan el mayor atraso social y económico del país, con altos índices de violencia, ocio, desempleo y drogadicción.

La alta vulnerabilidad social se manifiesta en varios aspectos como la pobreza, el desempleo, o bien trabajadores sin seguridad social o con un empleo de baja calidad, un trabajo mal remunerado, comunidades con bajos niveles de educación y malas condiciones de salud. Se puede ampliar el concepto de alta vulnerabilidad social cuando se visualiza la existencia de barreras curriculares, materiales, humanas y ambientales, donde los problemas sociales que las afectan tienen un rango de alcance que va más allá de la comarca inmediata (González, 2005).

Por tanto, se consideran como zonas de alta vulnerabilidad social los asentamientos irregulares de personas que migran del campo a la ciudad en busca de mejores situaciones de vida, pero que al llegar se encuentran sin trabajo, sin dinero y sin un lugar propio donde vivir. Una gran cantidad de personas se establece en terrenos de manera ilícita, en casas construidas con latas y pedazos de madera, sin piso y sin los servicios básicos de drenaje y alcantarillado.

Ahora bien, al definirse zonas de alta vulnerabilidad como las comunidades urbano marginales situadas en lugares catalogados, por el gobierno costarricense, como de atención prioritaria, se hace necesario precisar qué se entiende por escuelas de este tipo. El Programa para el Mejoramiento de la Calidad de la Educación y Vida en las Comunidades Urbanas de Atención Prioritaria (PROMECUM) (González, 2005) las define como centros educativos integrales, no como instituciones aisladas, sino como centros comunitarios donde los estudiantes, además de aprender las materias del currículo, son educados y atendidos con respecto a los problemas sociales que los afectan fuera de la escuela, pero que influyen en su desempeño académico. Por tanto, el estudiante se visualiza como una persona que trae consigo un bagaje personal y social, que condiciona su desempeño académico.

\section{Metodología}

El estudio se sustentó en un enfoque cualitativo, según Barrantes (2001), en concordancia con la naturaleza del problema planteado y con los objetivos, por considerar que este es un primer acercamiento con el tema de inserción laboral en el campo de la docencia, en contexto de alta vulnerabilidad social. Se puede definir, de acuerdo con Kinnear y Taylor (2001), como un estudio que entrelaza lo exploratorio y lo descriptivo, al buscar sustento para proponer cambios en las 
condiciones en que se lleva a cabo el proceso de inserción laboral de los docentes, con el fin de ampliar los períodos de permanencia y de fortalecer su compromiso en este tipo de escuelas. Se espera, a la vez, que esta sea una estrategia para lograr no solo el bienestar de los estudiantes, sino también el de los mismos educadores y de la comunidad educativa en general.

Además, es de tipo aplicativo, ya que se espera que los hallazgos contribuyan a ampliar el nivel de conocimiento y a descubrir la necesidad de implementación de procesos de inserción laboral docente en la dinámica de los centros educativos, en este caso, en particular, en contextos que se caracterizan por un alto nivel de vulnerabilidad social.

Para la recolección y construcción de los datos se contó con el valioso aporte de la directora de la institución y de un grupo de 10 maestros, de los cuales cinco no tenían más de dos años de permanencia en la escuela y los otros cinco tenían más de seis años de laborar en esta.

Los instrumentos para la recolección de información utilizados fueron la entrevista semi-estructurada y el test de frases inconclusas, respectivamente.

La organización y el análisis de la información se efectuaron a partir de la definición de categorías y sub categorías; posteriormente, se procedió al diseño de matrices. Esto facilitó el análisis y la triangulación mediante un control cruzado entre el referente teórico, las opiniones de la directora y las expresiones de actitudes, creencias y sentimientos de los docentes.

Las categorías y sub-categorías definidas fueron: concepto de alta vulnerabilidad social que poseen los docentes; caracterización social, profesional y actitudinal de los docentes; proceso de inserción laboral y factores que influyen en la labor del personal docente en un proceso de inserción laboral desglosado en: contexto, familia y gestión administrativa. Como categorías emergentes surgieron el proceso de inserción laboral y otros factores.

\section{Principales hallazgos}

- La investigación, de acuerdo con las opiniones de la directora y las expresiones de los docentes, pone de manifiesto la inexistencia de procesos formales e intencionados de inserción laboral en la escuela en donde se realizó la experiencia. La directora justifica esta carencia, porque considera que el conocimiento requerido debió haberse dado en la formación académica. Con respecto a los docentes, ellos ratifican la necesidad de la implementación de estos procesos, siempre y cuando sean flexibles y contemplen componentes teórico- prácticos.

- Al contrastar las opiniones de los investigados con el referente teórico, se dan coincidencias con los autores Vonk (1996) y González et al. (2005), en cuanto a la inexistencia, en América Latina, de este tipo de procesos que permitan orientar la inserción laboral de los docentes en estos centros o en otros contextos. Tanto los autores como los docentes reiteran que no existen programas de acompañamiento a la inserción profesional. No obstante, González et al. (2005) agregan que, contrario a la realidad latinoamericana, algunos países europeos sí implementan programas de inducción formalmente establecidos.

- En relación con el concepto de alta vulnerabilidad social, los resultados de la investigación evidencian que tanto la directora como los docentes poseen una vaga noción, ya que no logran construir una definición en la cual se incorporen todas las características e implicaciones del término. Visualizan aspectos claves como son la existencia del riesgo social y enfrentar una serie de problemáticas como la pobreza, la delincuencia, la drogadicción, el abandono, la agresión, el abuso, la problemática familiar 
y un bajo nivel escolar. De ello podría inferirse que las personas en estudio poseen ideas aisladas, definiciones no consensuadas, fundamentadas en criterios y vivencias personales, por encima de una construcción colectiva teórico-práctica del concepto de alta vulnerabilidad social.

- A partir de la información suministrada por los docentes y por la directora, acerca de las nociones que poseen los profesionales que laboran en centros educativos de alta vulnerabilidad social en relación con las características sociales, se infiere que, a pesar de que existe un bagaje amplio de referente teórico sobre este aspecto, los docentes y, especialmente, la directora no expresan con claridad cuáles son las características sociales que debería poseer un docente para apoyar su trabajo en estos contextos. Inclusive, las respuestas de los docentes se centran en describir estrategias que promueven las interacciones sociales entre los estudiantes y mencionan las características de esas interacciones y no de sus propias habilidades sociales. Por su parte, la directora destaca la mística y el deseo de realizar las labores bien, las cuales no son características sociales sino que podrían considerarse como aspectos actitudinales.

- En cuanto al reconocimiento de las características actitudinales, tanto los docentes como la directora se refieren a ellas con mucha propiedad; las describen con precisión y les dan gran importancia; especialmente, a aquellas que facilitan las interacciones entre los diferentes actores sociales de la comunidad educativa. Entre estas actitudes resaltan: la orientación hacia el reforzamiento de la autoestima, la capacidad de interactuar con el medio y el interés por conocer la comunidad y la familia; también se refleja la convicción de los docentes de que con su accionar deben convertirse en agentes de cambio. Por consiguiente, esto conlleva la responsabilidad de promover actitudes que refuercen o fomenten una construcción adecuada de la autoestima de los estudiantes, la cual tiende a debilitarse por las dinámicas sociales existentes en su realidad cotidiana.

- En cuanto a las características profesionales se encuentran que el personal que labora en este Centro Educativo tiene un alto nivel académico (únicamente hay dos bachilleres universitarios, pero cuentan con una amplia experiencia laboral, 26 y 10 años de servicio, respectivamente; ocho licenciadas, y la directora que es máster en Administración Educativa) lo cual podría considerarse clave para una experiencia exitosa. Sin embargo, algunos docentes destacan que el éxito de su inserción laboral ha dependido de la relación existente entre su formación universitaria y sus experiencias prácticas en diversos contextos. Lo anterior pone de manifiesto que ellos visualizan que la formación académica en estos contextos no es suficiente para consolidar la inserción laboral.

- Las opiniones, tanto de los docentes como de la directora, coinciden en que el conocimiento de las realidades de las familias y del contexto comunal es uno de los factores que influye en la permanencia del personal docente en centros educativos, ubicados en las zonas de alta vulnerabilidad social. Además, enfatizan que una forma de mejorar esta relación es la consolidación de un trabajo en equipo que promueva relaciones e interacciones positivas entre los actores sociales del proceso educativo.

- Otro factor relacionado con la permanencia del personal docente, analizado en el estudio, es la gestión administrativa. Los docentes reafirman las posiciones teóricas planteadas por Antúnez y Carnicero (2009) y González (2005), respecto de que la responsabilidad de un buen funcionamiento de la institución está en sus manos y que ello incide en la permanencia del personal docente en centros educativos, ubicados en las zonas de alta vulnerabilidad 
social. Como parte de este buen funcionamiento, se contempla la organización de una estrategia de inserción laboral que vaya más allá de la inducción (etapa inicial) y la ejecución de actividades durante el curso lectivo.

- Entre otros factores que emergen, y que tanto los docentes como la directora consideran influyentes en el proceso de inserción laboral en estos centros educativos, se citan como elementos claves para el crecimiento personal la colaboración entre colegas y el desarrollo de la capacidad de escucha.

\section{Propuesta para la inserción laboral de docentes en contextos de alta vulnerabilidad social}

La propuesta para la inserción laboral se desarrolla en una escuela de Atención Prioritaria de la provincia de Heredia con un alto nivel de vulnerabilidad social, con el propósito de apoyar procesos de inserción laboral en otros centros del país. Dicha proposición surge como respuesta a la inexistencia de procesos de inserción laboral señalados por docentes, y apoyados en el referente teórico e institucional, fruto de la investigación.

\section{Lineamientos y estrategias}

Con el propósito de apoyar procesos de inserción laboral, en contextos de alta vulnerabilidad social, se plantean a continuación algunos lineamientos y estrategias que orienten su implementación. Cabe mencionar que, si bien es cierto, a lo largo de la investigación los docentes visualizan que la función de mediación la debe desarrollar el director, esto no tiene que ser indispensable; por el contrario, podría considerarse la posibilidad de que en los centros educativos exista un comité que organice y oriente su implementación.

\section{Lineamientos}

1. Diseñar y poner en práctica estrategias de inserción laboral que progresivamente consoliden un modelo institucional apoyado en el referente teórico y como respuesta concreta a las necesidades de cada centro educativo.

2. Construir un concepto de alta vulnerabilidad social entre los docentes y la dirección, el cual oriente la labor institucional, la mediación pedagógica y las relaciones entre toda la comunidad educativa.

3. Favorecer procesos de auto-reflexión que permitan definir con claridad qué entiende el personal docente por características sociales que él debe poseer. Identificarlas a nivel teórico, autoevaluarse individualmente y analizar en forma grupal cuáles poseen, o bien, cuáles se requieren para un adecuado desempeño en contextos de alta vulnerabilidad social.

4. Valorar y enriquecer las características actitudinales que les han ayudado a consolidar experiencias exitosas y, a través de ese intercambio, lograr la potencialización en ellos de cada una de estas, al hacer énfasis en la necesidad de reforzar actitudes que fortalezcan la autoestima en los niños y las niñas. 
5. Generar espacios de relaciones e interacciones positivas para favorecer la comprensión entre la familia, la comunidad y la escuela y que, a su vez, ayuden al docente a fortalecer las características profesionales que van más allá del conocimiento teórico logrado en su formación académica.

6. Implementar, por parte de la gestión administrativa, un proceso de inserción laboral que incluya: la inducción (etapa inicial), la ejecución de actividades durante el curso lectivo y la capacitación de docentes, de acuerdo con las demandas reales. Este proceso debe promover, como estrategia para fortalecer la permanencia laboral en la institución, el crecimiento personal y profesional mediante el trabajo colaborativo.

\section{Estrategias}

* En relación con el establecimiento de un proceso de inserción laboral: la gestión administrativa o algún otro facilitador, que puede entenderse como una comisión o comité, debe contemplar como parte de sus funciones la definición de algunas estrategias de inserción laboral que visualice, como una primera fase, la inducción; posteriormente, la ejecución de actividades durante el curso lectivo y, como aspecto complementario, la capacitación, en la cual se tomen en cuenta las demandas reales del docente.

Por ello, para cada fase se propone lo siguiente:

1. Fase de inducción: Se espera que esta fase sea ejecutada al inicio del curso lectivo, que tenga como objetivo que el docente, aunque sea nuevo, se sienta integrado y parte de un equipo de trabajo, el cual se consolidará en la medida en que conozca los lineamientos institucionales y el contexto geográfico, social, cultural y económico de la zona en donde se desarrolla la experiencia educativa. Entre las actividades mínimas que se contemplan para esta primera fase se encuentran:

a. Presentación del personal y dinámica sobre las expectativas profesionales y particulares de cada docente.

b. Recopilación de nociones previas que posee el docente sobre la comunidad, las familias y el entorno donde se encuentra inmerso el centro educativo.

c. Descripción de las responsabilidades y de los retos específicos que debe asumir un docente en una escuela de atención prioritaria.

d. Distribución del personal por niveles, contemplando la existencia de al menos un maestro con experiencia en el contexto para cada grado o ciclo.

e. Entrega en versión digital del diagnóstico comunal e institucional para la lectura individual y una posterior puesta en común con el grupo de docentes. 
f. Presentación oral de los programas específicos con los que cuenta la institución; entre estos se destacan: el Programa de Recuperación Integral del Niño y la Niña (Prinn), las tutorías, la forma de asignación de los recargos, así como las funciones y responsabilidades del equipo interdisciplinario.

g. Realización de un recorrido por la comunidad.

h. Reconstrucción de las percepciones obtenidas al realizar el recorrido por la comunidad, para evitar estereotipos errados de la realidad social existente.

i. Recomendaciones por parte de los docentes con más años de experiencia en la institución, sobre el manejo adecuado de la comunicación con la familia y de la disciplina con los niños.

2. Fase de implementación de actividades durante el curso lectivo: Esta segunda fase pretende generar un proceso de acompañamiento que se caracterice por el apoyo constante del colectivo que integra la comunidad educativa, una vez que el docente ya se encuentra inmerso en la cotidianeidad de la realidad y las implicaciones que tiene el trabajo en comunidades de alta vulnerabilidad social. Por tanto, esta fase no se limita a un tiempo específico, sino que se desarrolla a lo largo de todo el curso lectivo. Dentro de esta fase se incluye la realización de capacitaciones docentes de acuerdo con las necesidades que, como grupo, presenten análisis y actualización del POA; tarde de intercambio de experiencias exitosas de aula; desarrollo de sesiones de reflexión; organización, en conjunto con algún miembro del equipo interdisciplinario, de actividades específicas con familias que les ayuden a mitigar o superar situaciones conflictivas; establecimiento de tiempos para el fomento de la recreación y de las manifestaciones artísticas con todos los miembros del personal. Otra actividad pertinente en esta fase podría ser la re-lectura de la monografía institucional y su socialización, para conocer el diagnóstico comunal, institucional, la reseña histórica y los datos fundamentales y que, de ser necesario, se realice su actualización.

En esta segunda fase se sugiere la organización de actividades extracurriculares, establecidas de manera conjunta entre la comunidad-escuela-padres de familia. Estas actividades pueden incluir: festivales deportivos, exhibición de talentos, participación en celebraciones patrias o días especiales como el día de la familia, de las personas adultas mayores, o bien actividades más académicas que capacitan, a los distintos miembros del núcleo familiar, para comprender los procesos cognitivos de los estudiantes, como pueden ser: charlas sobre el manejo de conducta, elaboración de material didáctico, talleres pedagógicos que promuevan el aprendizaje colectivo niñofamiliar, entre otros.

3. Fase de capacitación que responda a las demandas reales de los docentes: En esta fase se pretende dar una respuesta real a las situaciones emergentes que surjan en la dinámica institucional y que ayuden al docente a mejorar las dinámicas e interacciones existentes. Algunas estrategias sugeridas para esta fase son: 
- La estrategia de la telaraña, a partir de una actividad de integración grupal; luego de realizar un proceso de diagnóstico de los niños y un acercamiento a la comunidad y a las familias, más o menos un mes después de iniciado el curso lectivo, el facilitador o mediador de la actividad solicitará a los docentes que definan un interés, una motivación o una necesidad de capacitación que haya surgido, y esté latente en ellos, a partir de su incorporación a la institución. Con el objetivo de recabar insumos para la planificación de capacitaciones y el proceso de actualización, durante la dinámica se sistematizará la experiencia.

- Otra estrategia que se propone es el establecimiento de espacios de intercambios formales o informales, a partir de experiencias sencillas, pero innovadoras que hayan generado éxito en el desempeño de su función docente, con el objetivo de que estos espacios produzcan motivación y renovación profesional. Parte de estos espacios fueron descritos en la segunda fase del proceso de inducción.

Cabe mencionar que las estrategias planteadas en cada una de las fases pueden ser modificadas y enriquecidas, en tanto se visualice la inserción como un proceso que no es rígido ni estático.

Desde la visión de un proceso de inserción que responda a la necesidad de mejoramiento continuo, se propone evaluar las estrategias implementadas a partir de un FODA que permita, de ser necesario, cambiar, modificar o incluir nuevas estrategias de acuerdo con las necesidades y demandas surgidas de su puesta en marcha.

A continuación se enumeran algunas otras estrategias que pueden contribuir a fortalecer la propuesta de inserción laboral:

* En relación con la necesidad de establecer un concepto de alta vulnerabilidad social: Realizar una lluvia de ideas sobre el concepto personal que posee cada docente y la directora y, a partir de todos los aportes planteados, crear un nuevo concepto consensuado, que responda a la realidad que se vive en la comunidad.

* En relación con la dificultad para definir características sociales del docente: Facilitar un test que incluya un listado de características sociales, de manera que a nivel individual el docente realice, en primera instancia, un proceso de auto-reflexión y, posteriormente, en grupo determinen acciones para ampliarlas y fortalecerlas.

Para valorar la presencia de los criterios se ofrece una escala de calificación que permita al docente reconocer la manifestación de esa característica en su experiencia profesional. Este ejercicio se complementa con el establecimiento de compromisos individuales y grupales que constituyan una adecuada respuesta a los resultados obtenidos en el test. 


\section{TEST DE CARACTERÍSTICAS SOCIALES}

Marque con una equis (X) el nivel de vivencia personal que usted tiene, en relación con cada una de las características sociales que se le plantean a continuación y que pueden ayudarle a desenvolverse mejor en comunidades educativas de alta vulnerabilidad social.

\begin{tabular}{|c|c|c|c|}
\hline Característica social que usted posee & Mucho & Poco & Nada \\
\hline - Dinámico (a) & & & \\
\hline - Sociable & & & \\
\hline - $\quad$ Seguro (a) de sí mismo (a) & & & \\
\hline $\begin{array}{l}\text { - } \quad \text { Auto-controlado (a) } \\
\text { - } \quad \text { Buen ánimo }\end{array}$ & & & \\
\hline - Capacidad de comunicar a los estudiantes el entusiasmo por el aprendizaje & & & \\
\hline - Capacidad de trabajar con otras personas & & & \\
\hline $\begin{array}{l}\text { - Habilidad para establecer relaciones propositivas con los demás, dentro de } \\
\text { la escuela y la comunidad. }\end{array}$ & & & \\
\hline - Habilidad para mantener relaciones interpersonales armoniosas & & & \\
\hline - Desarrollar un estilo de trabajo en colaboración con los colegas & & & \\
\hline $\begin{array}{l}\text { - Disposición para adaptarse a las necesidades de una situación y cambiar } \\
\text { de táctica }\end{array}$ & & & \\
\hline - Habilidad de fijar expectativas y parámetros claros & & & \\
\hline $\begin{array}{l}\text { - Habilidad para entender a otras personas y tratar de comprender por qué } \\
\text { se comportan de la forma como lo hacen }\end{array}$ & & & \\
\hline $\begin{array}{l}\text { - Trabajar con los padres para aprender más sobre sus hijos y ayudarles a } \\
\text { comprenderlos }\end{array}$ & & & \\
\hline $\begin{array}{l}\text { - Colaboración con otras personas para llegar a objetivos comunes respecto } \\
\text { del aprendizaje estudiantil }\end{array}$ & & & \\
\hline - Establecer reglas de convivencia social & & & \\
\hline - $\quad$ Promover tratos justos y equitativos & & & \\
\hline - Crear relaciones cálidas y valorativas con sus estudiantes & & & \\
\hline $\begin{array}{l}\text { - Mantener vínculos de colaboración con sus padres, con las familias y con } \\
\text { los miembros de la comunidad }\end{array}$ & & & \\
\hline
\end{tabular}

Con el objetivo de precisar el compromiso real que el docente adquiere con el desarrollo de este tipo de características, se procede a dejar una evidencia escrita de este proceso, por medio de un cuadro que registre cada una de las características que se desea reforzar a nivel individual y para cada una de ellas establecer el compromiso personal para su logro. 
Una vez realizada la experiencia anterior, se procede en colectivo a establecer una serie de compromisos grupales que faciliten el fortalecimiento de dichas características.

* En relación con la necesidad de valorar y enriquecer las características actitudinales de los docentes: Se propone crear un espacio de socialización en donde, a partir de una pregunta generadora, cada docente comparta una actitud personal que le haya facilitado el trabajo con niños dentro de esta comunidad, debe procurar referirse a actitudes diversas, de manera que se fortalezca la importancia de desarrollar múltiples actitudes proactivas y contemplando la importancia de fomentar la autoestima de los estudiantes. Una vez concluida la experiencia, se socializará el listado de actitudes que, a partir de la experiencia integradora, ellos mismos consideraron como fundamentales y, a nivel grupal, decidirán si es necesario incluir alguna nueva que haya surgido a través de la dinámica.

* En relación con la necesidad de promover la colaboración entre colegas y desarrollar la capacidad de escucha como elementos claves para el crecimiento personal: Se proponen espacios de intercambios formales o informales, como son convivios y tardes académicas o conversatorios, entre otros. Con esto se pretende que a partir de experiencias sencillas e innovadoras, que hayan generado éxito en el desempeño de su función docente, se produzcan espacios de motivación y renovación profesional. Parte de estos espacios fueron descritos en la segunda fase del proceso de inducción.

A modo de reflexión final se enfatiza que si bien es cierto las investigadoras establecen un conjunto de lineamientos y estrategias que conforman una propuesta de inserción laboral, estas pueden ser modificadas, ya que solo constituyen un primer intento y el punto de partida para la construcción de un modelo de inserción laboral docente, que responda a las realidades de los diferentes contextos educativos. Pero se espera que los docentes, al incorporarse al sistema educativo en diferentes contextos, se concienticen de la necesidad de establecer procesos que les permita acercarse a la realidad, conocer sus características, su problemática. Además, que definan estrategias de trabajo cooperativo, las cuales con el tiempo irán siendo mejoradas, reestructuradas y enriquecidas, con el fin de generar espacios óptimos para el bienestar de todos los actores sociales, quienes desempeñan un papel activo dentro de los contextos de alta vulnerabilidad social.

\section{Referencias bibliográficas}

Antúnez, S. y Carnicero, P. [Coords.). (2009). Modelo regional de gestión escolar en Centroamérica y República Dominicana: Análisis de la situación y propuestas para la convergencia regional. Universidad de Barcelona: Colección IDER.

Arce, A. I., Gamboa, G., Glock, D., Paitel, P. y Sánchez, M. (2001). Propuesta: Proyecto de creación de centros educativos bilingües francés-español en la enseñanza pública costarricense: etapa experimental. San José, Costa Rica: Ministerio de Educación Pública, Asesoría de Francés. Recuperado de http://www.mep.go.cr/CentroDeInformacion/DOC/12\%20\%20Proyecto\%20 de\%20creación\%20de\%20centros\%20educativos\%20bilingüies\%20fra-303200985030.pdf 
Barrantes, R. (2001). Investigación: un camino al conocimiento un enfoque cualitativo y cuantitativo. San José, Costa Rica: EUNED.

Consejo Superior de Educación. (1995). Política Educativa hacia el Siglo XXI. San José, Costa Rica: Ministerio de Educación Pública.

González, A., Araneda, N., Hernández, J. y Lorca, J. (2005). Inducción profesional docente. Estudios pedagógicos, 31(1), 51-62. doi: 10.4067/S0718-07052005000100003

González, A. (2005) Promecum: Educación contra la pobreza en Costa Rica. En C. Ornelas (Coord.), Buenas prácticas de educación básica en América Latina (Tomo I), (288-300). México, ILCE. Recuperado de http://es.scribd.com/doc/4875276238/38/Promecum-Educacion-contrala-pobreza-en-Costa-Rica

Kinnear, T. y Taylor, J. (2001). Investigación de mercados: un enfoque aplicado (4ª ed.). México. Editorial McGraw-Hill.

Peralta, V. (2007). Una pedagogía de las oportunidades: Nuevas ventanas para los párvulos latinoamericanos del siglo XXI. Santiago de Chile: Andrés Bello.

Programa Estado de la Nación en Desarrollo Humano Sostenible. (2008). Estado de la Educación (2). San José, Costa Rica: CONARE.

Sojo, A. (2003, agosto). Vulnerabilidad social, aseguramiento y diversificación de riesgos en América Latina y el Caribe. Revista de la CEPAL 80, 121-140. Recuperado de http://www. eclac.cl/publicaciones/xml/7/19367/lcg2204e-Sojo.pdf

Vonk, J. H. C. (1996). A Knowledge Base for Mentors of Beginning Teachers: Results of a Dutch Experience. En R. McBridge (Ed.). Teacher Education Policy. Some Issues Arising from Research and Practice (pp. 113-134). London: Falmer Press. 